\title{
Similarity Solutions of Unsteady Convective Boundary Layer Flow along Isothermal Vertical Plate with Porous Medium
}

\author{
Md. Yeakub Ali, Mohammed Nasir Uddin, Md. Jasim Uddin, N. M. Ridwan Zahed \\ Department of Mathematics, Chittagong University of Engineering \& Technology, Chittagong, Bangladesh \\ Email: ali69cuet@gmail.com
}

Received 1 December 2015; accepted 25 December 2015; published 30 December 2015

Copyright (C) 2015 by authors and Scientific Research Publishing Inc.

This work is licensed under the Creative Commons Attribution International License (CC BY).

http://creativecommons.org/licenses/by/4.0/

(c) (i) Open Access

\begin{abstract}
Similarity solution of unsteady convective boundary layer flow along isothermal vertical plate with porous medium is analyzed. The plate surface is reactive with the fluid and generates inert specie which diffuses inside the boundary. The flux of the specie at the plate is proportional to specie concentration at the plate. The governing equations of continuity, momentum, energy and specie diffusion are transformed into ordinary differential equation by using the similarity transformation and solved numerically by using free parameter method along with shooting technique. The dimensionless velocity, temperature and concentration profiles are obtained and presented through figures for different parameters entering into the problem. The local Skin-friction co-efficient, Nusselt number and Sherwood number at the plate for physical interest are also discussed through tables.
\end{abstract}

\section{Keywords}

Similarity Solution, Unsteady Flow, Porous Medium, Convective Boundary Layer Flow, Vertical Plate

\section{Introduction}

The problem of unsteady free convective boundary layer flow has attracted the interest of many investigators in view of its application in many engineering and geophysical problems. Over the years studies involving the boundary layer flow along reactive surface have grown to researchers due to its implicit occurrence in different engineering and natural applications like melt spinning, contamination transport from surfaces, acid mine drainage remediation, waste disposal, reactive flow of gases adjacent to the surface and so on. Fluid is also sprayed 
for cleaning the surface; the contamination at the surface reacts with the plate and washes away. In addition the processes of Micro Electro Mechanical System (MEMS) require deposition or selective removal of specie from the surface. Thus it has several utilities in micro or macro engineering, material science and environmental science, etc. In this context, Soundalgekar [1] studied the effect of mass transfer and free convection on the flow past an impulsively started vertical flat plate. Raptis et al. [2] constructed similarity solutions for boundary layer near a vertical surface in a porous medium with constant temperature and concentration. Many transport processes exist in nature and in industrial applications in which the simultaneous heat and mass transfer occur as a result of combined buoyancy effects of thermal diffusion and diffusion of chemical species. Bejan and khair [3] reported on the natural convection boundary layer flow in a saturated porous medium with combined heat and mass transfer. Williams et al. [4] studied the unsteady free convection flow over a vertical flat plate under the assumption of variations of the wall temperature with time and distance. They found possible solution for a variety of classes of wall temperature distribution. The similarity solutions of free, mixed and forced convection problems in a saturated porous media were discussed by Ramanaiah et al. [5]. Watanabe [6] considered the mixed convection boundary layer flow past an isothermal vertical porous flat plate with uniform suction or injection. Kafoussias and Williams [7] investigated the effect of temperature-dependent viscosity on free-forced convective laminar boundary layer flow past a vertical isothermal plate. Erikson et al. [8] examined heat and mass transfer on a moving continuous flat plate with suction or injection on the flat plate. Slaouti et al. [9] studied the unsteady free convection flow in the stagnation point region of a three-dimensional body. It was seen that temperature and surface heat transfer were changed in a small interval of time. The surface heat transfer parameter increased with the increase of Prandtl number while the surface skin friction parameter decreased with the increase of Prandtl number. Healmy K. A. [10] studied MHD unsteady free convective boundary layer flow past a vertical plate embedded in a porous medium. Pop and Postelnicu [11] obtained similarity solutions of free convection boundary layers over vertical and horizontal surface porous media with internal heat generation. Chamkha and Khaled [12] obtained similarity solutions for hydro magnetic mixed convection heat and mass transfer for Hiemenz flow through porous media. Hossin and Monir [13] analyzed a two dimensional mixed convection flow of a viscous incompressible fluid of temperature dependent viscosity past a vertical plate. Seddek and Salem [14] studied the laminar mixed convection flow adjacent to vertical continuously stretching sheets with variable viscosity and thermal diffusivity. Aydin and Kaya [15] studied mixed convection of a viscous dissipating fluid about a vertical flat plate. They assumed that the fluid thermal conductivity was supposed to vary as a linear function of temperature. Aziz [16] investigated a similarity solution for laminar thermal boundary layer over a flat plate with a convection surface boundary condition. Ali and Magyari [17] studied the problem for unsteady stretching surface under different conditions by using a similarity method to transform governing time-dependent boundary layer equations into a set of ordinary differential equations. Mukhopadhyay [18] obtained similarity solution for unsteady mixed convection flow past a stretching sheet considering the combined effects of porous medium and the thermal radiation. Vajravelu [19] studied unsteady convective boundary layer flow of a viscous fluid at a vertical surface with variable fluid properties. Singh and Sharma [20] analyzed heat and mass transfer in the boundary layer flow along a vertical isothermal reactive plate near stagnation point.

The present problem is to study the similarity solutions of unsteady convective boundary layer flow along isothermal vertical plate with porous medium. Mentioned fluid flow, heat and mass transfer models are parameterized by dimensionless quantities such as Prandtl number, Schmidt number, unsteadiness and Buoyancy parameters, etc. The effect of governing parameters on the velocity, temperature, concentration profile are investigated and analyzed. The local Skin-friction coefficient, Nusselt number and Sherwood number at the plate for physical interest are also illustrated for required parameters.

\section{Governing Equations}

We consider an unsteady two dimensional flow of a viscous incompressible fluid through porous media along vertical plate. The flux of the specie at the plate is considered proportional to specie concentration at the plate. All the fluid properties assumed to be constant except the effect of buoyancy and the density variation. In addition the electric field, pressure gradient, Hall effects, joule heating terms and induced magnetic field are also neglected. Let the $x$-axis be taken along the direction of the plate and $y$-axis normal to it. If the velocity distribution in the potential flow is given by $U_{\infty}(x)=a$ and $V_{\infty}(y)=a y$, where a is a positive constant. Under assump- 
tions mentioned above, the governing boundary layer equations of continuity, momentum, energy and specie concentration can be written as:

$$
\begin{gathered}
\frac{\partial u}{\partial x}+\frac{\partial v}{\partial y}=0 \\
\frac{\partial u}{\partial t}+u \frac{\partial u}{\partial x}+v \frac{\partial u}{\partial y}=\gamma \frac{\partial^{2} u}{\partial y^{2}}+g \beta\left(T-T_{\infty}\right)+g \beta *\left(C-C_{\infty}\right)+U_{\infty} \frac{d U_{\infty}}{d x}-\frac{\gamma}{\tilde{\kappa}}\left(u-U_{\infty}\right) \\
\frac{\partial T}{\partial t}+u \frac{\partial T}{\partial x}+v \frac{\partial T}{\partial y}=\frac{\kappa}{\rho C_{p}} \frac{\partial^{2} T}{\partial y^{2}} \\
\frac{\partial C}{\partial t}+u \frac{\partial C}{\partial x}+v \frac{\partial C}{\partial y}=D \frac{\partial^{2} C}{\partial y^{2}}
\end{gathered}
$$

The boundary condition for velocity, temperature and concentration fields are:

$$
\begin{aligned}
& y=0, u=0, v=0, T=T_{w}, C=C_{w} \\
& y=\infty, u \rightarrow U_{\infty}=a, T \rightarrow T_{\infty}, C \rightarrow C_{\infty}
\end{aligned}
$$

where $u$ and $v$ are the fluid velocity components along $x$ and $y$-axis respectively, $\gamma$ is the kinematics viscosity, $\rho$ is the density of the fluid, $\beta$ and $\beta^{*}$ are the thermal and concentration expansion co-efficient respectively, $g$ is the acceleration due to gravity, $T$ is the temperature inside the boundary, $T_{\infty}$ is the temperature for away from the plate, $C$ is the species concentration in the boundary layer, $C_{\infty}$ is the species concentration of the ambient fluid, $C_{p}$ is the specific heat, $D$ is the molecular diffusivity of the species concentration, $\tilde{\kappa}$ is the permeability of the porous medium. and $\kappa$ is the co-efficient of thermal conductivity.

In order to simplify the mathematical analysis of the problem we introduce the flowing dimensionless variables:

$$
\eta=y \sqrt{\frac{a}{\gamma}} \frac{1}{x t}, x=\xi, t=\tau \text { and } u=a f^{\prime} .
$$

Introducing dimensionless similarity variables into the system of Equation (2) to Equation (4) is reduced to system of ordinary differential equations;

$$
\begin{gathered}
f^{\prime \prime \prime}+A_{1} f f^{\prime \prime}+A_{2} \eta f^{\prime \prime}+G_{r} \theta+G_{c} \phi-K\left(f^{\prime}-1\right)=0 \\
\theta^{\prime \prime}+P_{r}\left(A_{1} f+A_{2} \eta\right) \theta^{\prime}=0
\end{gathered}
$$

and

$$
\phi^{\prime \prime}+S_{c}\left(A_{1} f+A_{2} \eta\right) \phi^{\prime}=0
$$

It is observed that the Equation (1) is identically satisfied.

The Boundary condition in Equation (5) is reduced to the corresponding boundary condition for velocity, temperature and concentration fields are as:

where

$$
\begin{aligned}
& f(0)=0, f^{\prime}(0)=0, \theta(0)=1, \phi(0)=1 \text { at } \eta=0 \\
& f^{\prime}(\infty)=1, \theta(\infty)=0, \phi(\infty)=0 \text { at } \eta=\infty .
\end{aligned}
$$

$G_{r} \quad($ Local Temperature Grashof number $)=\frac{g \beta \xi^{2} \tau^{2}}{a^{2}}\left(T_{w}-T_{\infty}\right)$,

$G_{c}$ (Local Concentration Grashof number) $=\frac{g \beta^{*} \xi^{2} \tau^{2}}{a^{2}}\left(C_{w}-C_{\infty}\right)$, 
$K \quad$ (Permeability parameter) $=\frac{\xi^{2} \tau^{2} \gamma}{a \tilde{\kappa}}$,

$S_{c}($ Schomidt number $)=\frac{\gamma}{D}$,

$P_{r} \quad$ (Prandtl number) $=\frac{\mu c_{p}}{\kappa}$

and Unsteadiness parameters $A_{1}=\xi \tau^{2}, A_{2}=\frac{\xi^{2} \tau}{a}$.

The above equations with boundary condition are solved numerically by using shooting method.

\section{Important Physical Parameters}

From the engineering point of view, the physical quantities of interest are the local Skin-friction coefficient $C_{f_{x}}$, local Nusselt number $\mathrm{Nu}_{x}$ and local Sherwood number $S_{h_{x}}$ respectively defined as

$$
\begin{aligned}
& C_{f}=\frac{\tau_{w}}{\rho U_{w}^{2} / 2}=2(\operatorname{Re})^{-1 / 2} f^{\prime \prime}(0), \\
& N u_{x}=\frac{x q_{w}}{k \Delta T}=-(\operatorname{Re})^{1 / 2} \theta^{\prime}(0), \\
& S h=\frac{x m_{w}}{D \Delta C}=-(\operatorname{Re})^{-1 / 2} \phi^{\prime}(0)
\end{aligned}
$$

where the wall shear stress $\tau_{w}$, the wall heat flux $q_{w}$ and the quantity of mass transfer $m_{w}$ through the unit area of the surface. These are given by

$$
\tau_{w}=\mu\left(\frac{\partial u}{\partial y}\right)_{y=0} ; q_{w}=k\left(\frac{\partial T}{\partial y}\right)_{y=0}, m_{w}=D\left(\frac{\partial C}{\partial y}\right)_{y=0}
$$

Thus the values of the local skin-friction co-efficient $C_{f_{x}}$, local Nusselt number $N u_{x}$ and local Sherwood number $S_{h_{x}}$ are proportional to $f^{\prime \prime}(0),-\theta^{\prime}(0)$ and $\phi^{\prime}(0)$ respectively as Vajravelu et al. [19].

\section{Numerical Solution}

Systems of ordinary differential equations together with boundary conditions are nonlinear and coupled. First of all, higher order non-linear differential Equations (7) to (9) are converted into simultaneous linear differential equations of first order and these are further transformed into initial value problem solved numerically by applying a standard initial value solver i.e., the Shooting method namely Nachtsheim-Swigert (1965) iteration technique (guessing the missing value) along with Runge-Kutta integration scheme.

In a shooting method, the missing (unspecified) initial condition at the initial point of the interval is assumed, and the differential equation is then integrated numerically as an initial value problem to the terminal point. The accuracy of the assumed missing initial condition is then checked by comparing the calculated value of the dependent variable at the terminal point with its given value. If a difference exists, another value of the missing initial condition must be assumed and the process is repeated. This process is continued until the agreement between the calculated and the given condition for the specified degree of accuracy.

In the process of iteration the Skin friction coefficient, the Nusselt number and the Sherwood number, are proportional to $f^{\prime \prime}(0),-\theta^{\prime}(0)$ and $\phi^{\prime}(0)$ also evaluated. The numerical result obtained for several selected values of the established parameter are displayed in figures and tables. These figures and tables show that velocity, temperature, concentration, rate of heat transfer and skin friction coefficient are affected significantly with the variations of the considered controlling parameters.

\section{Results and Discussions}

In order to get physical insight into the problem numerical solution have been carried out for different values of 
the considered thermo physical parameters of controlling the fluid dynamics in the flow regime. The value of the Prandtl number are taken to be $\mathrm{Pr}=0.71,01.00$ and 07.00 which correspond to air, electrolyte solution such as salt water and fresh water at $25^{\circ} \mathrm{C}$ and $1 \mathrm{~atm}$ pressure. The values of Schmidt number are chosen to be $\mathrm{Sc}=$ 00.22, 00.62 and 02.62 representing diffusing chemical species of most common interest in air like hydrogen, water vapor, and Propel Benzene respectively at $25^{\circ} \mathrm{C}$ and $1 \mathrm{~atm}$ pressure. Our attention is based on the positive values of the buoyancy parameter (Gr, Gc) in such way that Grashof number Gr corresponds to the cooling problem and solutal Grashof number Gc indicates the chemical species concentration in the free stream region is less than the concentration at the boundary surface. Here it is considered that the value of Suction parameter is 0.06. The cooling problem is often considered in engineering applications for example in the cooling of electronic components and nuclear reactors. For the purpose of discussing the result, the numerical calculations are presented in the form of non dimensional velocity, temperature and concentration.

\subsection{Velocity Profiles}

The effects of various parameters on the non dimensional velocity profiles in the boundary layer are depicted in Figures 1-9. Generally it is observed in these figures that fluid velocity is lowest at the plate surface and increases gradually to its free stream values far away from the plate surface satisfying the boundary condition for all parameters. Figures 1-4 reveal the effects of unsteadiness parameters $A_{1}$ and $A_{2}$ on the dimensionless velocity.

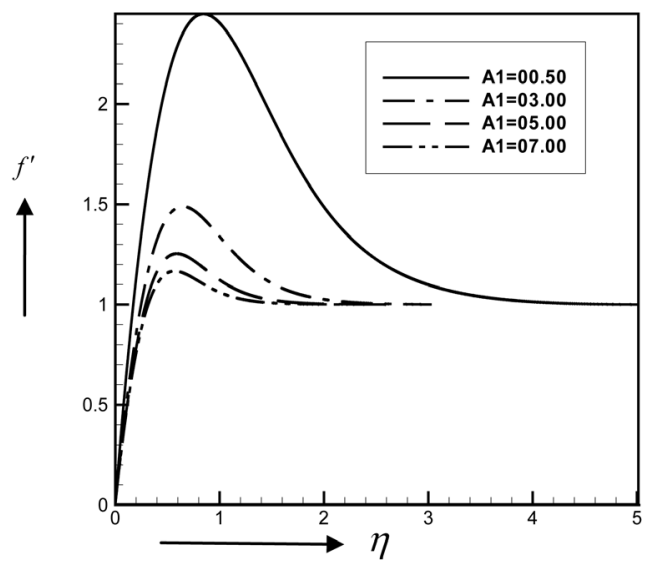

Figure 1. Velocity profile for various values of $A_{1}$ when, $\mathrm{A}_{2}=0.50, \mathrm{Pr}=0.71, \mathrm{Sc}=0.22, \mathrm{Gr}=05.0, \mathrm{Gc}=$ $05.00, \mathrm{~K}=00.60$.

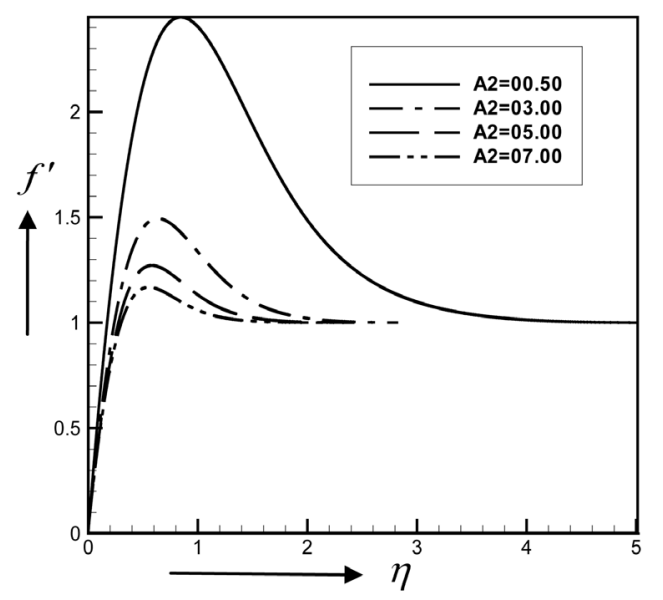

Figure 2. Velocity profile for various values of $A_{2}$ when, $\mathrm{A}_{1}=0.50, \mathrm{Pr}=00.71, \mathrm{Sc}=0.22, \mathrm{Gr}=05.00$, $\mathrm{Gc}=05.00, \mathrm{~K}=0.60$. 


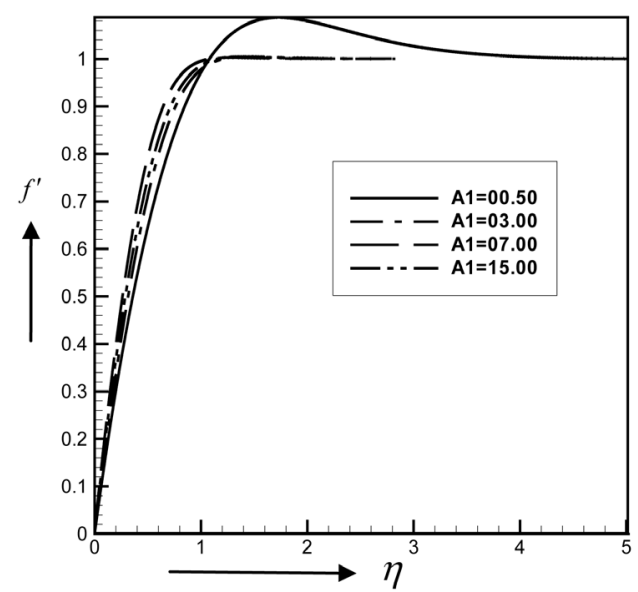

Figure 3. Velocity profile for various values of $A_{1}$ when $\mathrm{A}_{2}=0.50, \operatorname{Pr}=0.71, \mathrm{Sc}=0.22, \mathrm{Gr}=00.50$, Gc $=0.50, \mathrm{~K}=0.60$.

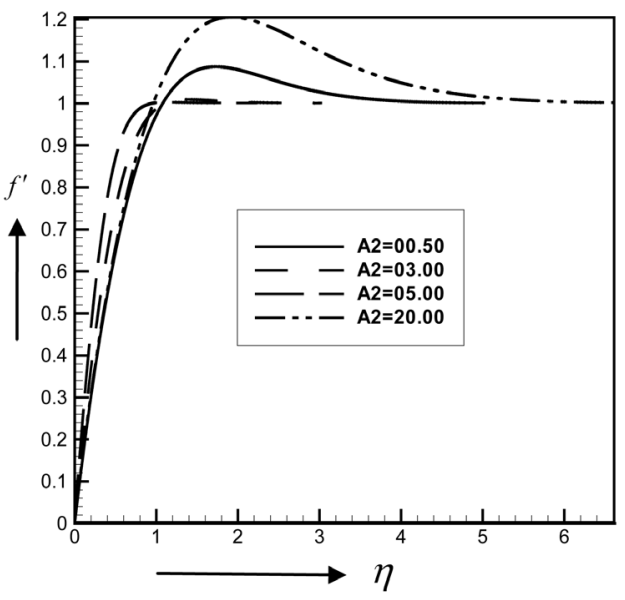

Figure 4. Velocity profile for various values of $\mathrm{A}_{2}$ when $\mathrm{A}_{1}=0.50, \mathrm{Pr}=00.71, \mathrm{Sc}=0.22, \mathrm{Gr}=05.00, \mathrm{Gc}$ $=05.00, \mathrm{~K}=0.60$.

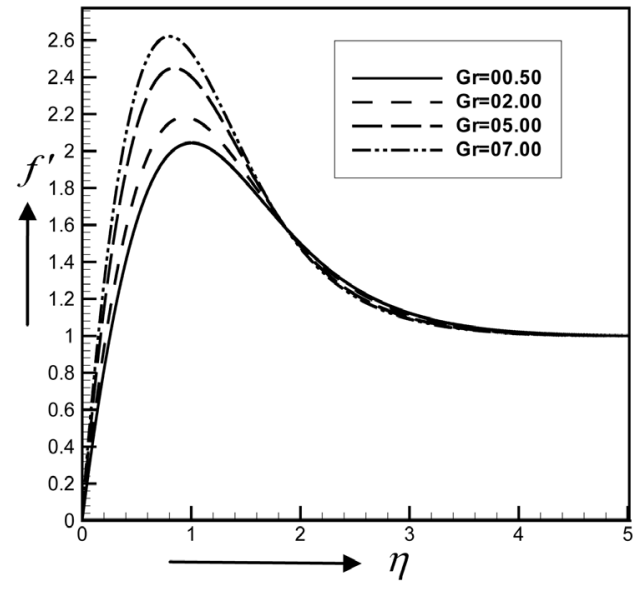

Figure 5. Velocity profile for various values of $\mathrm{Gr}$ when $\mathrm{A}_{1}=0.50, \mathrm{~A}_{2}=0.50, \mathrm{Pr}=0.71, \mathrm{Sc}=00.22$, Gc $=05.00, \mathrm{~K}=00.60$. 


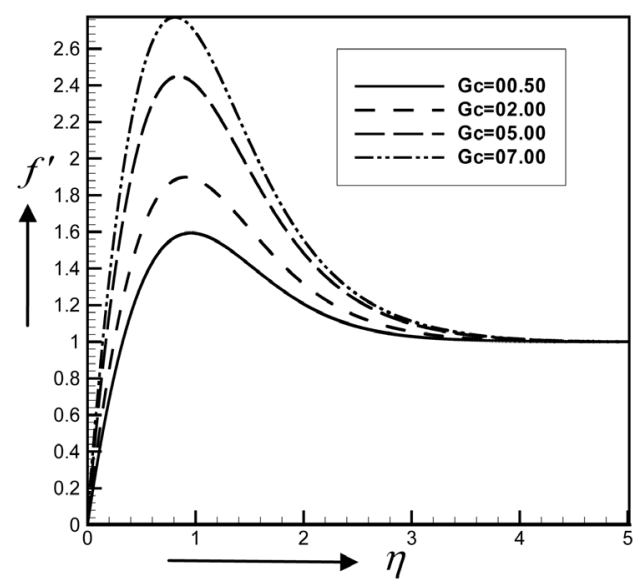

Figure 6. Velocity profile for various values of Gc when $\mathrm{A}_{1}=0.50, \mathrm{~A}_{2}=0.50, \operatorname{Pr}=00.71, \mathrm{Sc}=00.22, \mathrm{Gr}$ $=05.00, \mathrm{~K}=0.60$.

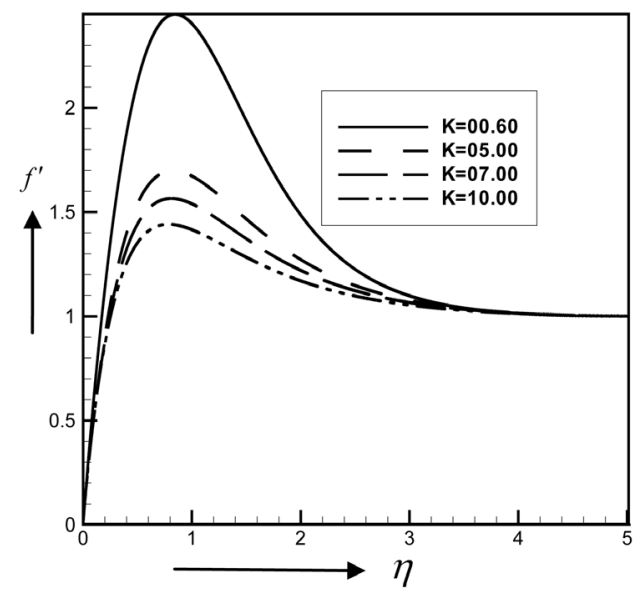

Figure 7. Velocity profile for various values of $\mathrm{K}$ when $\mathrm{A}_{1}=0.50, \operatorname{Pr}=00.71, \mathrm{Sc}=0.22, \mathrm{Gr}=05.00, \mathrm{Gc}$ $=05.00, \mathrm{~K}=0.60$.

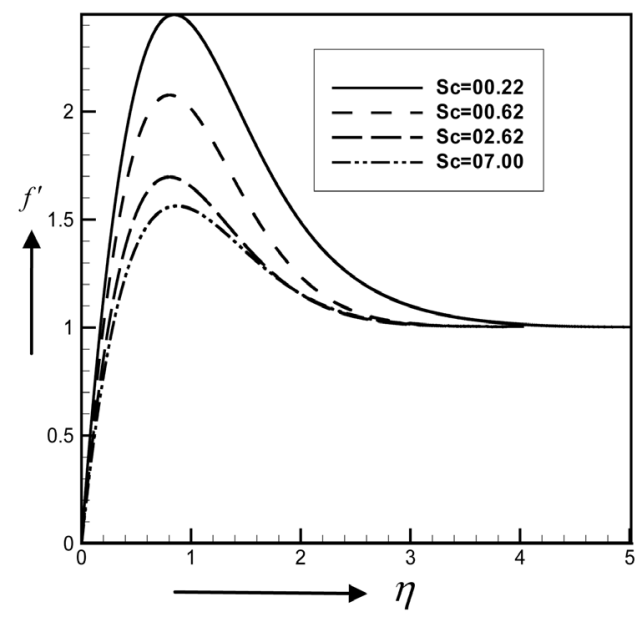

Figure 8. Velocity profile for various values of Sc when $A_{1}=00.50, A_{2}=00.50, P r=00.71, G r=05.00$, $\mathrm{Gc}=05.00, \mathrm{~K}=00.60$. 


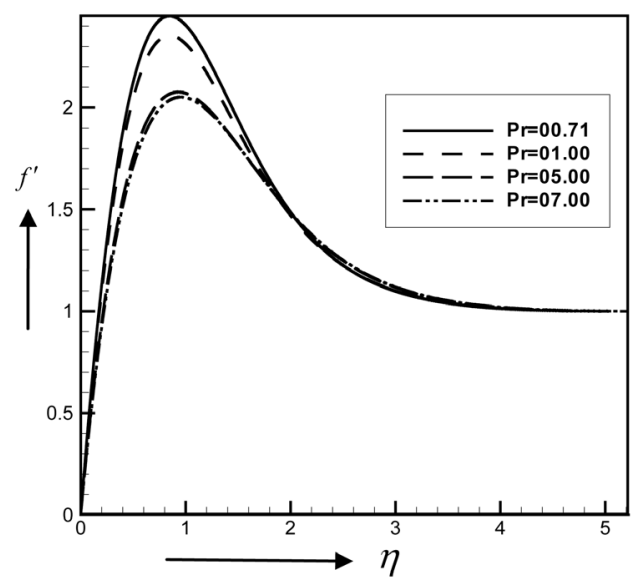

Figure 9. Velocity profile for various values of Pr When $\mathrm{A}_{1}=00.50, \mathrm{~A}_{2}=00.50, \mathrm{Sc}=00.22, \mathrm{Gr}=05.00, \mathrm{Gc}=$ $05.00, \mathrm{~K}=00.60$.

In Figures 1-3, it is observed that the velocity profile decreases with the increases of $A_{1}$ and $A_{2}$. Figure 4 depicts the velocity profile increases with the increases of $A_{2}$.

Figures 5-6 represent the variation of the velocity profiles with the buoyancy forces parameter ( $\mathrm{Gr}$ and $\mathrm{Gc}$ ). In both cases a downward acceleration of the fluid is observed with increasing intensity of buoyancy forces. Furthermore these figures show that increases in buoyancy forces leads to an increases in velocity profile. It is established that increase in buoyancy forces enhance the fluid flow.

Figure 7 display the velocity profile for selected values of permeability parameter K. It is noticed that velocity decreases for increasing values of $\mathrm{K}$ but far away from the plate the profiles overlap as shown in this figure.

The influence of the Schmidt number on the velocity profile is shown in Figure 8. It is found that the fluid velocity decreases rapidly with the increases of Schmidt number.

Figure 9 exhibits the effect of the Prandtl number on the non dimensional velocity profiles in the boundary layer. We observed that the velocity profile decreases with the increase of Pr, which is due to fact that as Pr increases the dynamic viscosity of the fluid increases which then slow down the velocity of the fluid.

\subsection{Temperature Profiles}

Figures 10-16 depict the behavior of the dimensionless temperature profiles for the effect of various thermo physical parameters.

As per the boundary conditions of the flow profile under consideration, the fluid temperature attain its maximum values at the plate surface and decreases exponentially to the free stream zero value far away from the plate.

Figures 10-11 illustrate the effects of unsteadiness parameters $A_{1}$ and $A_{2}$ on the dimensionless temperature. It is observed that in both cases the temperature profile decreases with the increases of $A_{1}$ and $A_{2}$ as expected.

Figures 12-13 represent the variation of the temperature profiles with the buoyancy forces parameter ( $\mathrm{Gr}$ and Gc). It is observed that in both cases the temperature boundary layer thickness decreases with an increase in the buoyancy forces parameter (Gr and Gc).

Figure 14 displays the temperature profile for selected values of permeability parameter $\mathrm{K}$. It is noticed that temperature profile slightly increases for increasing values K.

The influence of various values of the Schmidt number Sc on the dimensionless temperature profile is represented in Figure 15. We see that the fluid temperature profile increases monotonically with the increases of Schmidt number.

Figure 16 illustrates the effect of the Prandtl number on the non dimensional temperature profiles in the boundary layer. We observed that the temperature profile decreases smoothly with the increase of Pr.

\subsection{Concentration Profiles}

Effects of physical parameters variation on the chemical species concentration profiles are depicted in Figures 17-23. It is noteworthy that the chemical species concentration is highest at the plate surface and decreases 


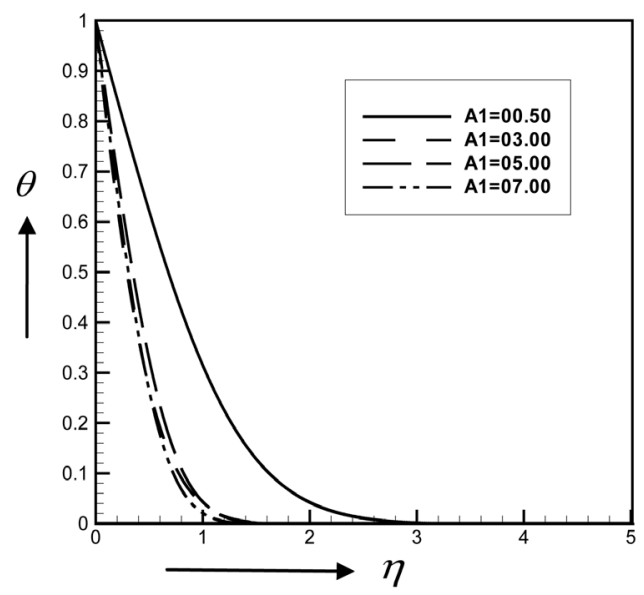

Figure 10. Temperature profile for various values of $A_{1}$ when $A_{2}=0.50, P r=00.71, S c=00.22, G r=05.00$, $\mathrm{Gc}=05.00, \mathrm{~K}=00.60$.

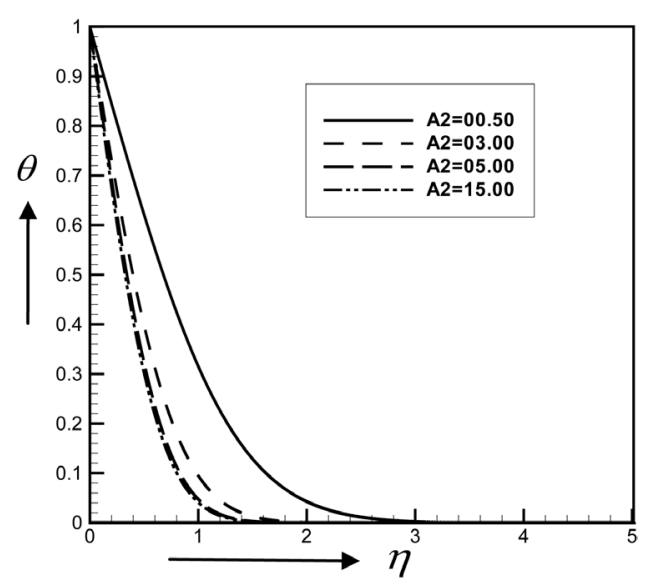

Figure 11. Temperature profile for various values of $\mathrm{A}_{2}$ when $\mathrm{A}_{1}=0.50, \mathrm{Pr}=00.71, \mathrm{Sc}=00.22 \mathrm{Gr}=05.00$, $\mathrm{Gc}=05.00, \mathrm{~K}=0.60$.

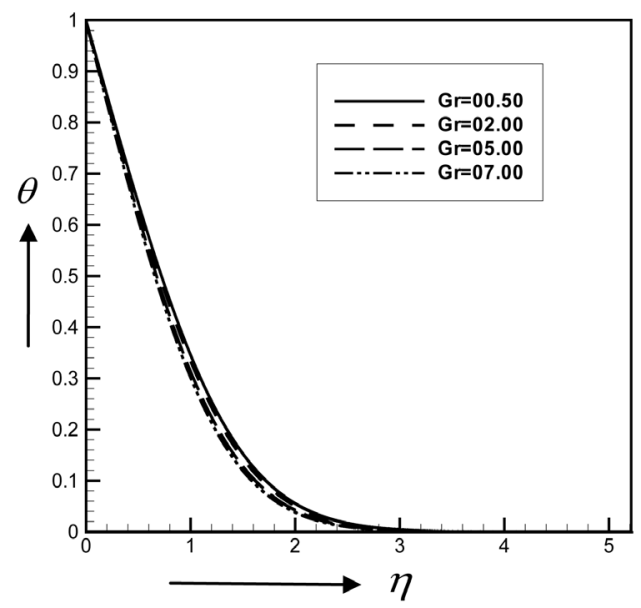

Figure 12. Temperature profile for various values of Gr when $\mathrm{A}_{1}=0.50, \mathrm{~A}_{2}=00.50, \mathrm{Sc}=00.22, \mathrm{Gc}=$ $05.00, \mathrm{Gc}=05.00, \mathrm{~K}=0.60$. 


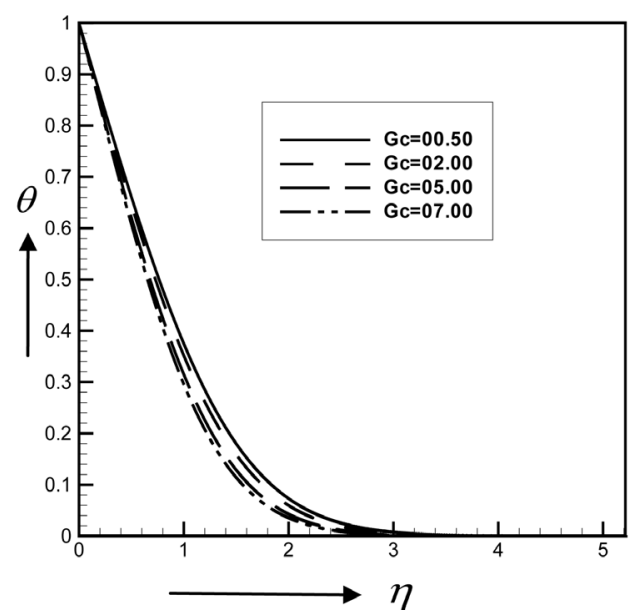

Figure 13. Temperature profile for various values of Gc when $\mathrm{A}_{1}=0.50, \mathrm{~A}_{2}=00.50, \mathrm{Sc}=00.22, \mathrm{Gr}=05$. $\mathrm{Pr}=00.71$. $\mathrm{K}=00.60$.

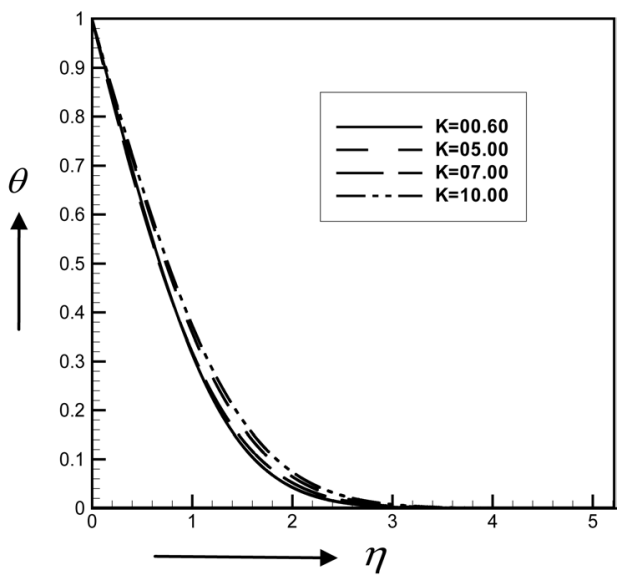

Figure 14. Temperature profile for various values of $\mathrm{K}$ when $\mathrm{A}_{1}=0.50, \mathrm{~A}_{2}=0.50, \mathrm{Sc}=00.22 \mathrm{Pr}=00.71$, $\mathrm{Gr}=05.00, \mathrm{Gc}=05.00$.

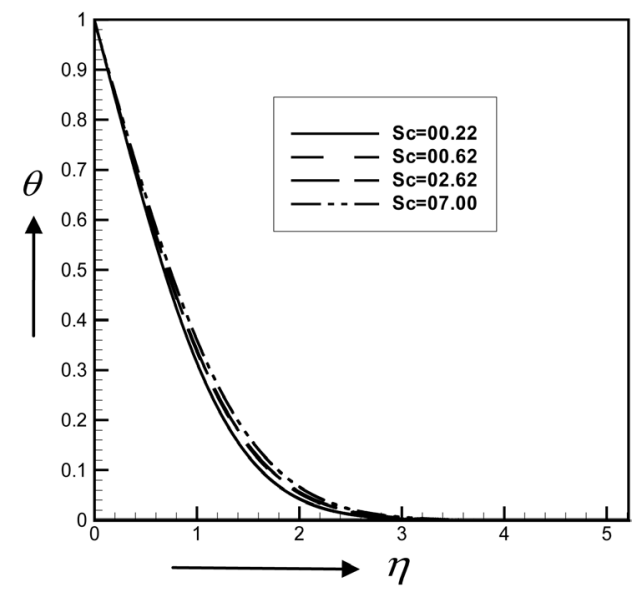

Figure 15. Temperature profile for various values of Sc when $A_{1}=0.50, A_{2}=0.50, \operatorname{Pr}=00.71, G c=05.00$, $\mathrm{Gr}=05.00, \mathrm{~K}=0.60$. 


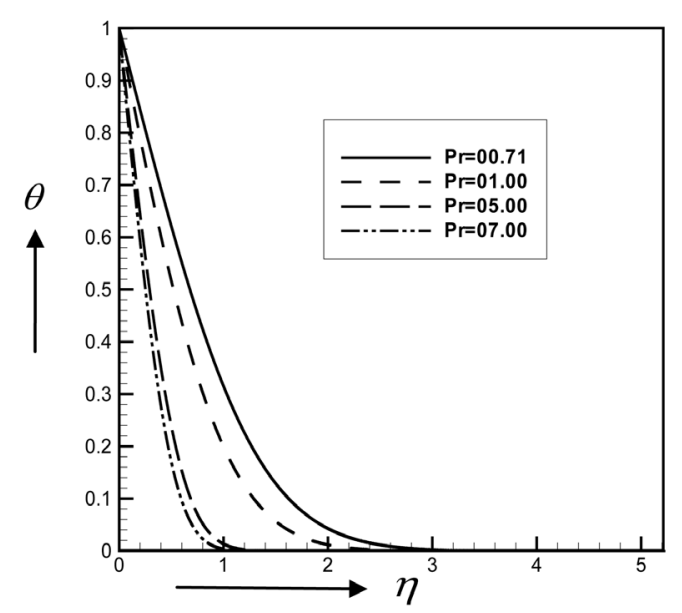

Figure 16. Temperature profile for various values of $\operatorname{Pr}$ when $A_{1}=$ $0.50, \mathrm{~A}_{2}=0.50, \mathrm{Sc}=00.22, \mathrm{Gr}=05.00, \mathrm{Gc}=05.00, \mathrm{~K}=0.60$.

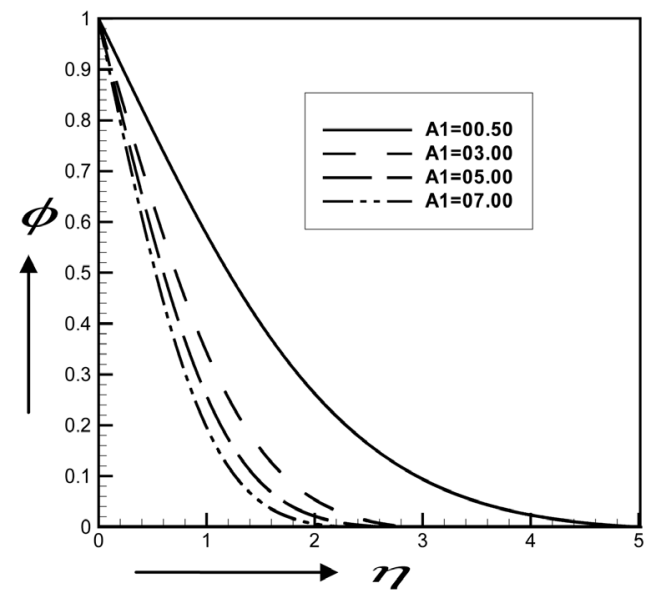

Figure 17. Concentration profile for various values of $\mathrm{A}_{1}$ when $\mathrm{A}_{2}=0.50, \mathrm{Pr}=00.71, \mathrm{Sc}=0.22 \mathrm{Gr}=05.00$, $\mathrm{Gc}=05.00, \mathrm{~K}=0.60$.

exponentially to zero far away from the plate satisfying the boundary conditions of the flow profile under consideration.

Figures 17-18 illustrate the effects of unsteadiness parameters $A_{1}$ and $A_{2}$ on the dimensionless concentration. It is observed that in both cases the concentration profile decreases with the increases of $A_{1}$ and $A_{2}$.

The influence of buoyancy parameters (Gr and Gc) on the concentration profile is illustrated in Figures 19-20. It is noticed that in both cases concentration boundary layer thickness slightly decreases with the increases in the buoyancy forces parameter ( $\mathrm{Gr}$ and $\mathrm{Gc}$ ). The decreasing in the concentration profile is not significant and near to coincide due to the increasing values of $\mathrm{Gr}$.

Figure 21 displays the concentration profile for selected values of permeability parameter K. It is noticed that concentration increases gradually for increasing values of $\mathrm{K}$.

The influence of selected values of the Schmidt number Sc on the dimensionless concentration profile is represented in the Figure 22. In this figure we see that the increasing value of The Schmidt number leads to decrease the concentration rapidly as expected. The Schmidt number embodies the ratio of the momentum diffusivity to the mass (Species) diffusivity. It physically relates the relative thickness of the hydrodynamics boundary layer and mass transfer (concentration) boundary layer.

Figure 23 illustrates the effect of the Prandtl number on the non dimensional concentration profiles in the boundary layer. We observed that the concentration profile decreases with the increase of Pr. 


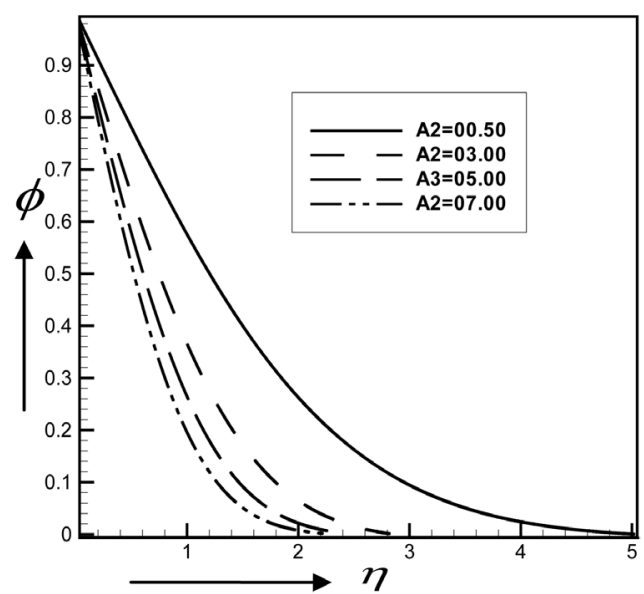

Figure 18. Concentration profile for various values of $\mathrm{A}_{2}$ when $\mathrm{A}_{2}=0.50, \mathrm{Pr}=0.71, \mathrm{Sc}=00.22 \mathrm{Gr}=05.00$, $\mathrm{Gc}=05.00, \mathrm{~K}=0.60$.

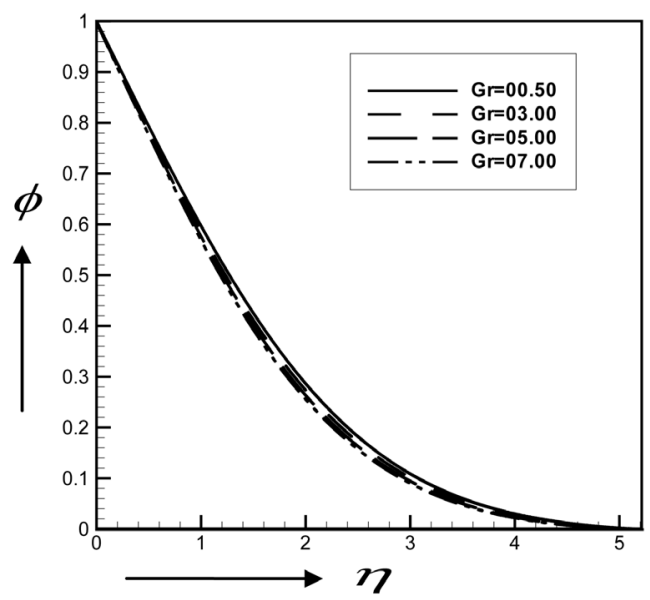

Figure 19. Concentration profile for various values of $\mathrm{Gr}$ when $\mathrm{A}_{1}=0.50, \mathrm{~A}_{2}=00.50, \mathrm{Pr}=00.71, \mathrm{Sc}=$ $00.22, \mathrm{Gc}=05.00, \mathrm{~K}=0.60$.

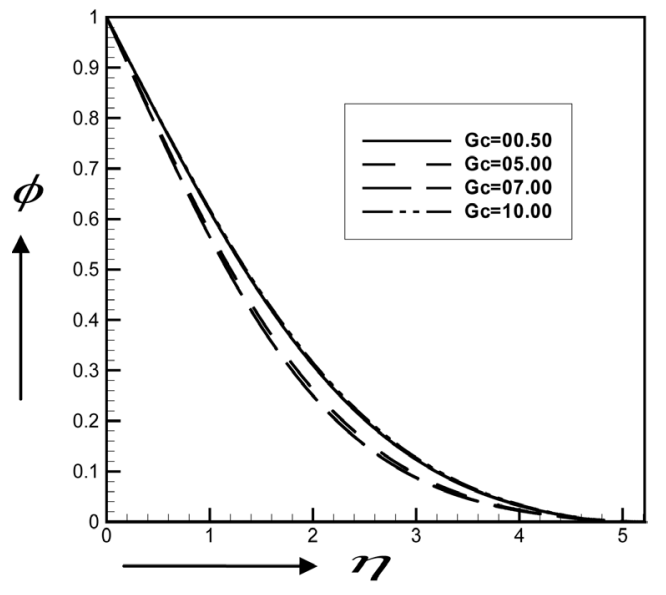

Figure 20. Concentration profile for various values of Gc when $\mathrm{A}_{1}=0.50, \mathrm{~A}_{2}=00.50, \mathrm{Pr}=00.71, \mathrm{Sc}=$ $00.22, \mathrm{Gr}=05.00, \mathrm{~K}=0.60$. 


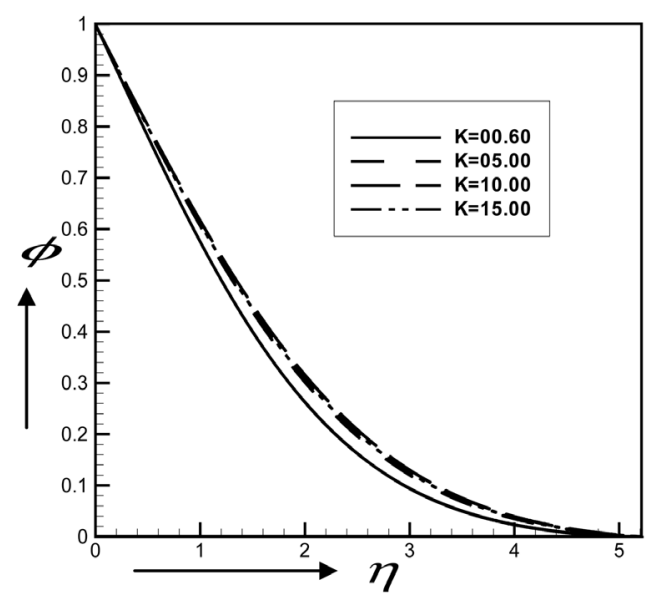

Figure 21. Concentration profile for various values of $\mathrm{K}$ when $\mathrm{A}_{1}=0.50, \mathrm{~A}_{2}=0.50, \mathrm{Pr}=00.71, \mathrm{Sc}=00.22$ $\mathrm{Gr}=05.00, \mathrm{Gc}=05.00$.

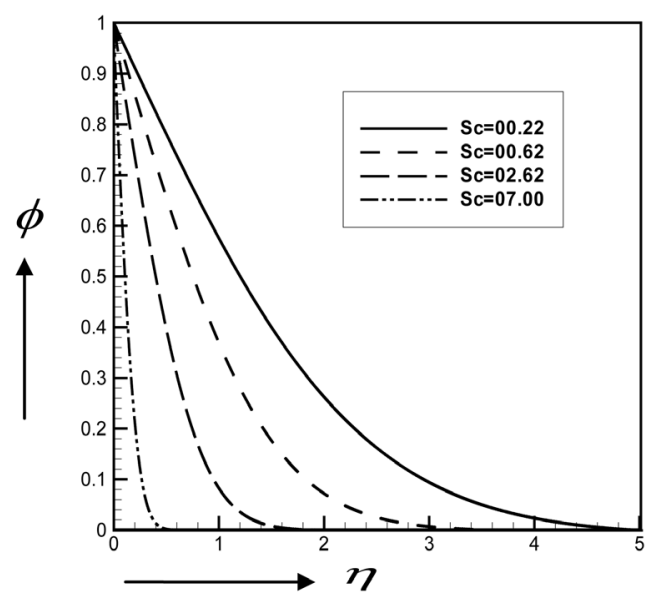

Figure 22. Concentration profile for various values of Sc when $A_{1}=0.50, A_{2}=00.50, \operatorname{Pr}=00.71, G r=$ $05.00, \mathrm{Gc}=05.00, \mathrm{~K}=0.60$.

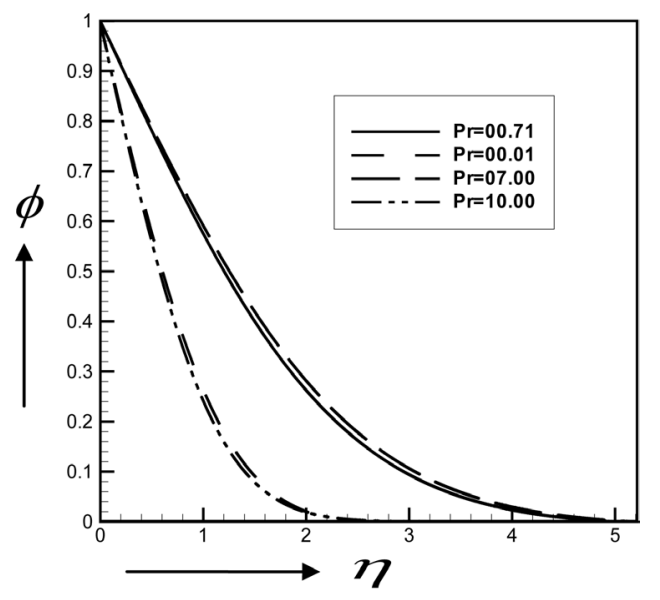

Figure 23. Concentration profile for various values of Pr when $A_{1}=0.50, A_{2}=0.50, S c=00.22, G r=05.00$, $\mathrm{Gc}=05.00, \mathrm{~K}=00.60$. 
Table 1. $f^{\prime \prime}(0), \theta^{\prime}(0)$ and $\phi^{\prime}(0)$ for different values of $\mathrm{A}_{1}$.

\begin{tabular}{cccc}
$\mathrm{A}_{1}$ & $f^{\prime \prime}(0)$ & $-\theta^{\prime}(0)$ & $-\phi^{\prime}(0)$ \\
\hline 00.50 & 6.6934176 & 0.78117126 & 0.4429248 \\
03.00 & 5.2826092 & 1.28027411 & 0.7400178 \\
05.00 & 5.0470532 & 1.53113842 & 0.8818370 \\
07.00 & 4.9614124 & 1.74274849 & 1.0033050 \\
\hline
\end{tabular}

Table 2. $f^{\prime \prime}(0), \theta^{\prime}(0)$ and $\phi^{\prime}(0)$ for different values of $\mathrm{A}_{2}$.

\begin{tabular}{cccc}
\hline $\mathrm{A}_{2}$ & $f^{\prime \prime}(0)$ & $-\theta^{\prime}(0)$ & $-\phi^{\prime}(0)$ \\
\hline 00.50 & 6.6934176 & 0.78117126 & 0.4429248 \\
03.00 & 5.4522855 & 1.26648094 & 0.7167768 \\
05.00 & 5.1329347 & 1.57378050 & 0.8849127 \\
07.00 & 5.0171984 & 1.83502576 & 1.0276074 \\
\hline
\end{tabular}

Table 3. $f^{\prime \prime}(0), \theta^{\prime}(0)$ and $\phi^{\prime}(0)$ for different values of Pr.

\begin{tabular}{cccc}
\hline $\operatorname{Pr}$ & $f^{\prime \prime}(0)$ & $-\theta^{\prime}(0)$ & $-\phi^{\prime}(0)$ \\
\hline 00.71 & 6.6934176 & 0.78117126 & 0.4429248 \\
01.00 & 6.5054144 & 0.90818887 & 0.4387351 \\
05.00 & 5.7397468 & 1.83812763 & 0.4252691 \\
07.00 & 5.6034713 & 2.13901438 & 0.4237254 \\
\hline
\end{tabular}

Table 4. $f^{\prime \prime}(0), \theta^{\prime}(0)$ and $\phi^{\prime}(0)$ for different values of Sc.

\begin{tabular}{cccc}
\hline Sc & $f^{\prime \prime}(0)$ & $-\theta^{\prime}(0)$ & $-\phi^{\prime}(0)$ \\
\hline 00.22 & 6.6934176 & 0.78117126 & 0.4429248 \\
00.62 & 6.0817840 & 0.74987414 & 0.7025626 \\
02.60 & 5.3109524 & 0.71578581 & 1.3287201 \\
07.00 & 4.8734894 & 0.70148763 & 2.0718250 \\
\hline
\end{tabular}

Table 5. $f^{\prime \prime}(0), \theta^{\prime}(0)$ and $\phi^{\prime}(0)$ for different values of Gr.

\begin{tabular}{cccc}
\hline $\mathrm{Gr}$ & $f^{\prime \prime}(0)$ & $-\theta^{\prime}(0)$ & $-\phi^{\prime}(0)$ \\
\hline 00.50 & 4.6873754 & 0.73448764 & 0.4206461 \\
03.00 & 5.8201508 & 0.76166186 & 0.4340122 \\
05.00 & 6.6934176 & 0.78117126 & 0.4429248 \\
07.00 & 7.5394318 & 0.79897511 & 0.4511300 \\
\hline
\end{tabular}

Table 6. $f^{\prime \prime}(0), \theta^{\prime}(0)$ and $\phi^{\prime}(0)$ for different values of Gc.

\begin{tabular}{cccc}
\hline Gc & $f^{\prime \prime}(0)$ & $-\theta^{\prime}(0)$ & $-\phi^{\prime}(0)$ \\
\hline 00.50 & 4.0141173 & 0.69600195 & 0.4002639 \\
03.00 & 5.5334838 & 0.74719038 & 0.4262365 \\
05.00 & 6.6934176 & 0.78117126 & 0.4429248 \\
07.00 & 7.8044404 & 0.81076762 & 0.4586696 \\
\hline
\end{tabular}


Table 7. $f^{\prime \prime}(0), \theta^{\prime}(0)$ and $\phi^{\prime}(0)$ for different values of $\mathrm{K}$.

\begin{tabular}{cccc}
$\mathrm{K}$ & $f^{\prime \prime}(0)$ & $-\theta^{\prime}(0)$ & $-\phi^{\prime}(0)$ \\
\hline 00.60 & 6.6934176 & 0.78117126 & 0.4429248 \\
03.00 & 5.9844309 & 0.73837860 & 0.4182700 \\
05.00 & 5.8250668 & 0.72020139 & 0.4081212 \\
07.00 & 5.8119254 & 0.70905409 & 0.4018858 \\
\hline
\end{tabular}

Finally, numerical results are illustrated in Tables 1-7 for the effect of various parameters on the skin-friction co-efficient, Nusselt number and Sherwood number. Table $1 \&$ Table 2 depict that with the increases of the unsteadiness parameters $A_{1} \& A_{2}$, skin-friction co-efficient decreases while Nusselt number and Sherwood number increases. It is shown in Table 3 that, with the increases of the Prandtl number (Pr), skin-friction co-efficient and Sherwood number decrease while Nusselt number increases. From Table 4, it is observed that with the increases of the Schmidt number (Sc), Skin friction coefficient and Nusselt number decreases while Sherwood number increases. Moreover, Tables 5-7 revel that skin-friction co-efficient increases with the increase of buoyancy forces ( $\mathrm{Gr}$ and $\mathrm{Gc}$ ) and decreases with the increases of the permeability parameter (K). It is also seen that Sherwood number increases with an increases of buoyancy forces and decreases with an increases of the permeability parameter $(\mathrm{K})$.

\section{Conclusion}

In this paper we have studied similarity solutions of unsteady convective boundary layer flow of viscous incompressible fluid along vertical isothermal plate. So far we know, experimental works for the problem are not evaluated ever. We are also expecting that using this solution the present study gives significant result for filling the gap in the existing literature. Numerical calculations are carried out for various values of the dimensionless parameters of the problem. It is concluded that the fluid velocity, temperature and concentration profiles decrease as the unsteadiness parameter increases. Both the Nusselt number and Sherwood number increase whereas Skin friction coefficient decreases with the increasing values of the unsteadiness parameter. The Nusselt number increases as the Prandtl number increases whereas Skin friction coefficient decreases with the increase of the Schmidt number (Sc) and Prandtl number (Pr). It is also revealed that velocity and specie concentration are higher at lower Schmidt number for low Prandtl fluid.

\section{Acknowledgements}

The authors are highly thankful to the authority of Chittagong University of Engineering \& Technology (CUET) for providing technical supports during this research work at Simulation Lab, Department of Mathematics, CUET, Chittagong, Bangladesh.

\section{References}

[1] Soundalgekar, V.M. (1979) Effect of Mass Transfer and Free-Convection on the Flow past an Impulsively Started Vertical Flat Plate. ASME Journal of Applied Mechanics, 46, 757-760. http://dx.doi.org/10.1115/1.3424649

[2] Raptis, A., Tzivanidis, G. and Kafousias, N.G. (1981) Free Convection and Mass Transfer Flow through a Porous Medium Bounded by an Infinite Vertical Limiting Layer Surface with Constant Suction. Letters in Heat and Mass Transfer, 8, 417-424.

[3] Bejan, A. and khair, K.R. (1985) Heat and Mass Transfer by Natural Convection in a Porous Medium. International Journal of Heat and Mass Transfer, 28, 909-918. http://dx.doi.org/10.1016/0017-9310(85)90272-8

[4] Williams, J.C., Mulligan, J.C. and Rhyne, T.B. (1987) Semi-Semilar Solutions for Unsteady Free-Convection Boundary Layer Flow on a Vertical Flat Plate. Journal Fluid Mechanics, 175, 309-332. http://dx.doi.org/10.1017/S0022112087000405

[5] Ramanaiah, G., Malarvizhi, G., et al. (1989) Unified Treatment of Similarity Solutions of Free, Mixed and Forced Convection Problems in a Saturated Porous Media. Proceedings of 6th International Conference on Numerical Problems, Swansea, 431-439.

[6] Watanabe, T. (1991) The Mixed Convection Boundary Layer Flow past an Isothermal Vertical Porous Flat Plate with Uniform Suction Or Injection. Acta Mech, 89, 123-132. http://dx.doi.org/10.1007/BF01171250 
[7] Kafoussias, N.G. and Williams, E.W. (1995) The Effect of Temperature-Dependent Viscosity on Free-Forced Convective Laminar Boundary Layer Flow past a Vertical Isothermal Plate. Acta Mechania, 110, 123-137. http://dx.doi.org/10.1007/BF01215420

[8] Erikson, L.E., Fan, L.T. and Fox V.G. (1996) Heat and Mass Transfer on a Moving Continuous Flat Plate with Suction or Injection on the Flat Plate. Industrial Engineering Chemical Fundamentals, 5, 19-25. http://dx.doi.org/10.1021/i160017a004

[9] Slaouti, A., Takhar. H.S. and Nath, G. (1998) Unsteady Free Convection Flow in the Stagnation-Point Region of a Three-Dimensional Body. International Journal of Heat and Mass Transfer, 41, 3397-3408. http://dx.doi.org/10.1016/S0017-9310(98)00080-5

[10] Healmy, K.A. (1998) Analyzed MHD Unsteady Free Convective Boundary Layer Flow past a Vertical Plate. ZAAM, 78, 255-270.

[11] Pop, I. and Postelnicu, A. (1999) Similarity Solutions of Free Convection Boundary Layers over Vertical and Horizontal Surface Porous Media with Internal Heat Generation. International Communications in Heat and Mass Transfer, 26, 1183-1191.

[12] Chamkha, A.J. and Khaled, A.A. (2000) Similarity Solutions for Hydromagnetic Mixed Convection Heat and Mass Transfer for Hiemenz Flow through Porous Media. International Journal of Numerical Methods for Heat \& Fluid Flow, 10, 94-115. http://dx.doi.org/10.1108/09615530010306939

[13] Hossain, Md.A. and Munir, Md.S. (2000) Mixed Convection Flow from a Vertical Flat Plate with Temperature Dependent Viscosity. International Journal of Thermal Sciences, 39, 173-183. http://dx.doi.org/10.1016/S1290-0729(00)00237-4

[14] Seddeek, M.A. and Salem, A.M. (2005) Laminar Mixed Convection Adjacent to Vertical Continuously Stretching Sheets with Variable Viscosity and Thermal Diffusivity. Heat and Mass Transfer, 41, 1048-1055. http://dx.doi.org/10.1007/s00231-005-0629-6

[15] Aydin, O. and Kaya, A. (2007) Mixed Convection of a Viscous Dissipating Fluid about a Vertical Flat Plate. Applied Mathematical Modelling, 31, 843-853. http://dx.doi.org/10.1016/j.apm.2005.12.015

[16] Aziz, A. (2009) A Similarity Solution for Laminar Thermal Boundary Layer over a Flat Plate with a Convection Surface Boundary Condition. Communications in Nonlinear Science and Numerical Simulation, 14, 1064-1068.

[17] Ali, M.E. and Magyari, E. (2007) Unsteady Fluid and Heat Flow Induced by a Submerged Stretching Surface While Its Steady Motion Is Slowed down Gradually. International Journal of Heat and Mass Transfer, 50, 188-195. http://dx.doi.org/10.1016/j.ijheatmasstransfer.2006.06.021

[18] Mukhopadhyay, S. (2009) Effect of Thermal Radiation on Unsteady Mixed Convection Flow and Heat Transfer over a Porous Stretching Surface in Porous Medium. International Journal of Heat and Mass Transfer, 52, 3261-3265. http://dx.doi.org/10.1016/j.ijheatmasstransfer.2008.12.029

[19] Vajravelu, K., Prasad, K.V. and Ng, C.-O. (2013) Unsteady Convective Boundary Layer Flow of a Viscous Fluid at a Vertical Surface with Variable Fluid Properties. Nonlinear Analysis: Real World Applications, 14, 455-464. http://dx.doi.org/10.1016/j.nonrwa.2012.07.008

[20] Singh, G. and Sharma, P.R. (2014) Heat and Mass Transfer in the Boundary Layer Flow along a Vertical Isothermal Reactive Plate near Stagnation Point. Journal of Applied Fluid Mechanics, 7, 25-33. 